\title{
Remote extradural hematoma after supratentorial brain tumor surgery: A rare complication of a routine
} surgery

\author{
Prashant Punia $M C h^{1}$ (D), Ashish Chugh $M C h^{2}$ iD, Sarang Gotecha $\mathbf{M C h}^{3}$ (iD) \\ 1,2,3 Department of Neurosurgery, Dr D Y Patil Medical College and Hospital, Pune, India
}

Date of submission: $22^{\text {nd }}$ May 2021

Date of acceptance: $17^{\text {th }}$ August 2021

Date of publication: $1^{\text {st }}$ September 2021

\section{Abstract}

Introduction: Hemorrhages in the surgical cavity are not uncommon and neurosurgeons are well versed with this entity. However, occurrence of an Extra Dural Hematoma (EDH) at a site remote to the surgical cavity, is rare. We review the literature and attempt to define and discuss the variables which play a role in determining the occurrence, extent and prognosis of the same.

Methods and Materials: The series includes three patients who developed post-operative EDH after excision of brain tumour in our hospital, from January 2017 to December 2019. All patients had a normal coagulation profile preoperatively. Data of these patients was examined closely in terms of age, sex, location, final diagnosis and treatment. Extensive review of literature was done and variables were drawn and compared based on the same.

Results: Out of a total of 601 patients operated for brain tumours, three patients $(0.5 \%)$ developed post-operative remote site EDH, two were males and one was female. The age range was 35 to 46 years with a mean of 41 years. Neither did any of these patients have a pre-existing hydrocephalus nor were they subjected to a CSF diversion procedure preoperatively. All three patients developed EDH on the ipsilateral side wherein two of our patients had a hematoma in the ipsilateral frontal region anterior to the surgical cavity while one patient developed hematoma in ipsilateral parietal region posterior to surgical cavity. Hematomas were unilateral with no extension to opposite side. Two patients had to be re-operated in an emergency setting while one patient was conservatively managed.

Conclusion: Remote EDH may develop in patients after removal of brain tumours and neurosurgeons must have a high index of suspicion for this entity

Key words: Extradural hematoma, Post-operative extradural hematoma, Remote extradural hematoma.

\section{Introduction}

$\mathrm{H}$ emorrhages in the surgical cavity after resection of tumor are not uncommon, however occurrence of an

Access this article online
Website: https://www.nepjol.info/index.php/NJN
DOI: https://doi.org/10.3126/njn.v18i3.37230
HOW TO CITE
Punia P, Chugh A. Remote extradural hematoma after
supratentorial brain tumor surgery: A rare complication of a
routine surgery. NJNS. 2021;18(3):55-60.

Address for correspondence:

Dr. Prashant Punia

Associate Professor, Department of Neurosurgery,

Dr. D.Y. Patil Medical College, Hospital and Research Center,

Sant Tukaram Nagar, Pimpri, Pune, India

E-mail: getdrprashant@gmail.com

Phone: +91-9049755538

Fax no: +91-20-27420439

Copyright (C 2021 Nepalese Society of Neurosurgeons (NESON)

ISSN: 1813-1948 (Print), 1813-1956 (Online)

This work is licensed under a Creative Commons

Attribution-Non Commercial 4.0 International License.
Extra Dural Hematoma (EDH), that too at a site remote to the surgical cavity, is rare ${ }^{1}$ and only a handful of incidences have been reported in the literature.

We studied occurrence of post-operative remote site $\mathrm{EDH}$ after tumor resection with review of literature and attempt to define and discuss the variables which play a role in determining the occurrence, extent and prognosis of the same.

\section{Methods and Materials}

We studied the admission from January 2017 to December 2019 who underwent brain tumor resection. Patients with deranged coagulation profile or patients on anticoagulation treatment were not operated upon until normalization of coagulation parameters. The data was examined closely in terms of age, sex, location, final diagnosis and treatment. Extensive review of literature was done and variables were drawn and discussed based on the same.

The tumour resection and EDH evacuation procedures were done at the same institute by the same surgical team and the standard procedures followed for both have been 


\section{Punia et al}

described below.

Tumor Resection: All patients were positioned either on a horseshoe head support or on a Mayfield's 3 pin head fixator. Utmost care was taken to prevent breach of inner table during positioning. One dose $(100 \mathrm{ml})$ of intravenous Inj. Mannitol 20\% (w/v) was administered at the completion of $1^{\text {st }}$ burr hole as a protocol. Dural hitch sutures using silk $4-0$, round bodied $20 \mathrm{~mm}$ needle were tacked up to the scalp before opening the dura. According to the site and extent of tumor, correct surgical approach was chosen and gross total excision was achieved. All significant neurovascular structures were preserved carefully. Excess Cerebro Spinal Fluid(CSF) drainage was countered by instilling warm Normal Saline (NS) into the surgical cavity. Meticulous hemostasis by coagulation of temporal artery stump and meningeal vessels was achieved using bipolar cautery and Surgicel®. Dural hitch sutures were placed along bone edges or against scalp in cases of a craniectomy. Dura was closed in a watertight fashion using an artificial dural graft wherever necessary. An epidural suction drain no. 14 was placed just prior to closure.

Extradural hematoma evacuation: Post-operatively, computed Tomography (CT) scan was done in all patients 6 hours after surgery as a part of an established department protocol and all patients with a finding of an EDH were assessed in terms of Glasgow Coma Scale(GCS) and were taken up for evacuation under emergency setting. Craniotomy for the same was performed according to the site of hematoma, as revealed by CT imaging. Post evacuation, dural hitch sutures were placed and precise hemostasis was achieved. Bone flap was not replaced and an epidural suction drain was placed post-operatively.

Post-operative course and follow up: The consciousness status was assessed periodically in the postoperative period and conventional symptomatic treatment was provided which was the same, irrespective of a first or second surgery. Post-operative follow up was telephonic, at the interval of 3 months.

\section{Results}

Of the 601 patients operated for brain tumors, three patients developed remote side EDH. Of which, two were male and one was female. The age range was 35 to 46 years with a mean of 41 years. The details are as in Table 1 .

None of the three patients have a pre-existing hydrocephalus nor were they subjected to a CSF diversion procedure preoperatively. Regarding tumor location, two of the tumors were located in right frontal region and one in right posterior parafalcine region. All three underwent a total tumor resection in supine position and their post-operative radiology reflected $\mathrm{EDH}$ on the routine postoperative scan done after 6 hours. All three patients developed EDH which were unilateral with no extension to opposite side. Two patients had to be re-operated in an emergency setting while one patient was conservatively managed. The time interval between tumor resection and recognition of hematoma was 6 hours in all cases. All patients had good recovery.

\section{Case Presentation} Pathology
35 years male

1 with 2 episodes of seizure
Parafalcine meningioma $5 \times 4 \times 4 \mathrm{~cm}$ (Figure 1). HPE: Meningothelial Meningioma grade-1
A large $4.2 \times 3 \times 2.8 \mathrm{~cm}$ sized heterogeneously enhancing

46-year-old

2 female with seizures tumour in the right frontal region with elevated choline peak on MR spectroscopy (Figure 2). HPE: Astrocytoma grade-3

\section{Events during hospital stay}

Simpson's grade-1 tumor resection. Routine 6 hours postoperative Scan showed large bi-convex hematoma in ipsilateral frontal region, far away from the tumour site. Urgent evacuation was done as his GCS started deteriorating. Post-operative course was uneventful and the patient was discharged on the 10th post-operative day without any neurological deficits.

Gross total resection of tumour was achieved and immediate post-operative period was uneventful.

A routine $\mathrm{CT}$ scan brain done after 6 hours revealed a large bi-convex EDH in the ipsilteral frontal region, away from the tumour bed, which was evacuated.

Post-operative course was uneventful and the patient was discharged on the 10th post-operative day without any neurological deficits.

A gross total resection of tumour was done. Routine CT done after 6 hours of surgery suggested a small bi-convex lesion in the ipsilateral parietal region, posterior to the site of surgery. contrast enhancing lesion with with recurrent headaches and

31 episode of peritumoural brain oedema causing mass effect (Figure 3). seizure in last 1 HPE: Low Grade Glioma week
The lesion was small and a decision to conservatively manage the same was taken. Patient didn't worsen during the course and was discharged on 7 th post-operative day.

Table 1: Details of patients with remote side extradural hematoma. 


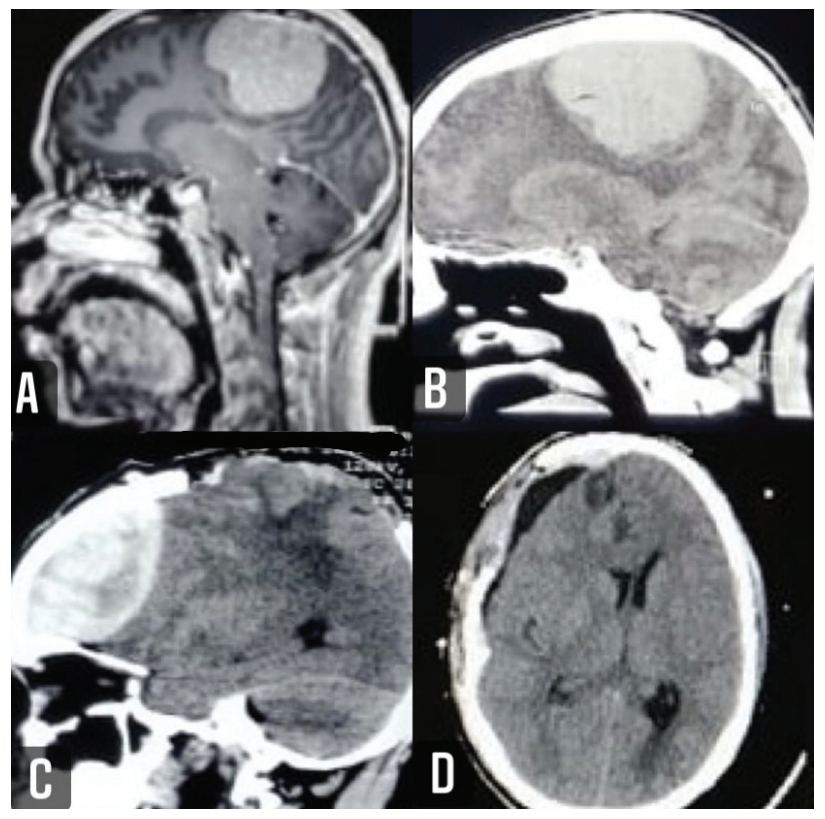

Figure 1: (A) Contrast enhanced T1 weighted Magnetic Resonance Imaging (MRI), sagittal view showing large dural based, homogeneously enhancing parasagittal lesion in right parietal region. (B) Contrast Enhanced Computed tomography (CECT) brain, sagittal view showing large dural based, homogeneously enhancing parasagittal lesion in right parietal region. (C) Postoperative Computed tomography (CT) brain, sagittal view showing complete excision of meningioma with craniectomy defect in parietal region. Also seen is a biconvex hyperdense lesion (EDH) in right frontal region. (D) Computed tomography (CT) brain, axial view after evacuation of EDH showing complete evacuation of EDH with extension of craniectomy in right frontal region.

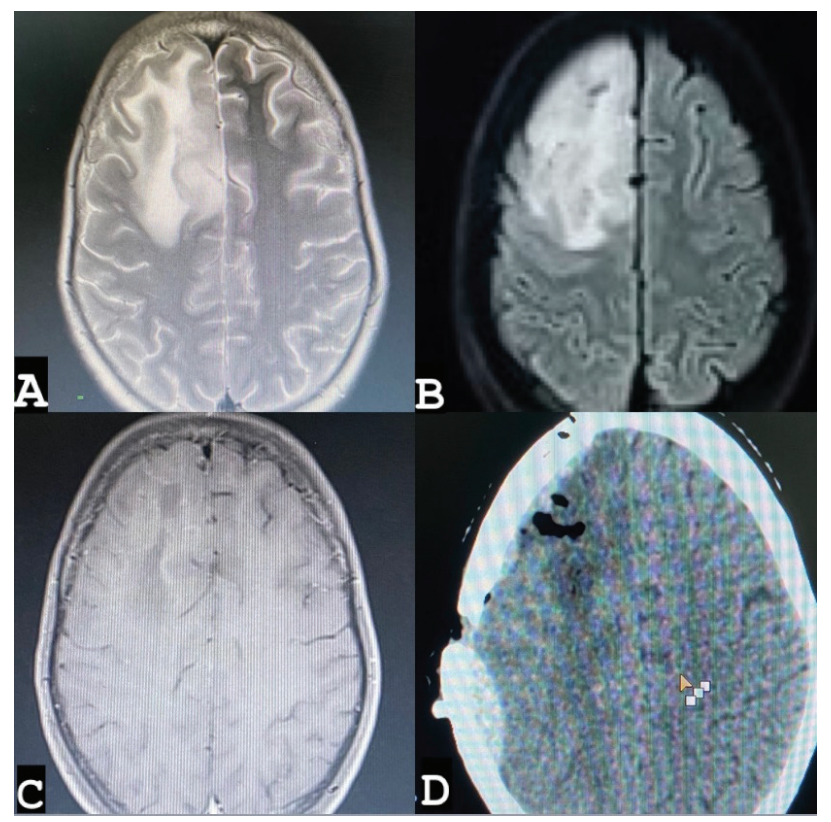

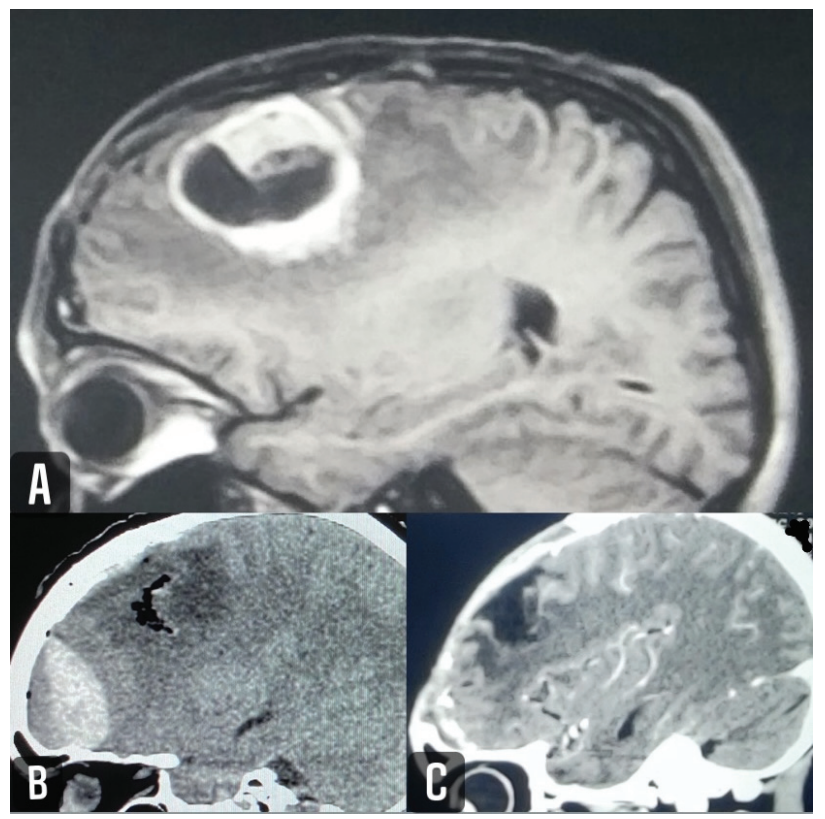

Figure 2: (A) Contrast enhanced T1 weighted Magnetic Resonance Imaging (MRI), sagittal view, showing a heterogeneously enhancing lesion in the anterior right parafalcine region. (B) Postoperative Computed tomography (CT) brain, sagittal view showing excision of lesion. Also seen is a biconvex hyperdense lesion (EDH) in right frontal region. (C) Contrast Enhanced Computed tomography (CECT) brain, sagittal view at 3 months follow up period showing frontal craniectomy site with complete evacuation of EDH and no recurrence of tumour.

Figure 3: (A) T2 weighted Magnetic Resonance Imaging (MRI), axial view, showing oedema with T2 hyperintense changes in right frontal region. (B) T2 FLAIR Magnetic Resonance Imaging (MRI), axial view, showing oedema with T2 hyperintense changes in right frontal region. (C) Contrast enhance T1 weighted Magnetic Resonance Imaging (MRI), axial view, showing non-enhancing, diffuse lesion in right frontal lobe. (D) Postoperative Computed tomography (CT) brain, axial view showing excision of lesion. Also seen is a biconvex hyperdense lesion (EDH) in right parietal region posterior to craniotomy limits of surgical site. 


\section{Discussion}

Intracranial hemorrhage is a common complication of craniotomy done for various reasons. Remote site $\mathrm{EDH}$, however, is a rare event and thus, has not been adequately reported in literature. In our series, only $0.5 \%$ ( 3 out of 601) patients developed this complication while Kalfas et al, ${ }^{2}$ in their survey of 4992 patients undergoing craniotomy, reported postoperative remote site EDH in 7 patinets $(0.0014 \%)$. These statistics indicate the rare nature of occurrence of remote postoperative EDH. The maximum rate of occurrence of postoperative EDH has been reported by Kim et al, ${ }^{3}$ who in their retrospective analysis of 941 patients undergoing craniotomy, reported $24(2.6 \%)$ patients with significant postoperative EDH. The relatively large rate of incidence may be due to the inclusion of patients in whom EDH occurred not only in the remote site but also in the operative cavity. The rare occurrence of remote postoperative EDH can be attributed to the almost universal craniotomy protocols to prevent postoperative hemorrhage, which include dural hitch sutures, meticulous hemostasis and leaving of extradural drains inside surgical cavity prior to closure.

The mean age of patients who developed remote postoperative EDH was 41 years with all 3 patients in our study being less than 50 years. Similar results have been published by Chung et al. ${ }^{4}$ All 3 of their patients were less than 30 years and in their extensive review of literature, authors found only 1 patient of more than 50 years who had developed remote postoperative EDH. Similar findings have been reported by other authors ${ }^{5}$ and it is safe to state that this entity is mainly seen in young and middle age group. The evident reason for the same is that the adhesions between dura and inner table of skull bone grows stronger as age progresses. Thus, it results in easy yielding of dura from the bone due to negative pressure, resulting in higher chances of EDH in young patients following intracranial tumor surgery. Location of the remote EDH is of great significance and can be classified into 4 types:

Type 1: hematomas that occur at the adjacent site of the ipsilateral surgical area

Type 2: hematomas that occur on the contra lateral side of surgical area

Type 3: hematomas involving bilateral sides

Type 4: supratentorial epidural hematomas after infratentorial surgery

All 3 patients in our study had type 1 hematoma based on location. The diagnosis of a postoperative hematoma plays a critical role in final outcome of the patient. There is a wide range of interval from tumor resection to hematoma occurrence and hematomas have been known to occur anytime between 30 minutes to 3 days. ${ }^{6}$ In our center, due to an established protocol, postoperative CT scan is done 6 hours after surgery or in an emergent setting post sudden deterioration of the patient, whichever is earlier. This protocol, while giving ample time for the hematoma to develop if it has to, leaves nothing to chance in case of an unpleasant radiological surprise. Hematoma was identified early in all 3 of our patients, two of whom were subjected to timely hematoma evacuation. This protocol was essentially and evidently helpful as it proved central to an early recognition of untoward outcome i.e. hematoma in the absence of any symptoms and timely management of the same. Death rates for EDH reported before the advent of CT was between $50-55 \%$ due to diagnostic delay and this makes the entity a ticking time bomb unless diagnosed early. Thus, it is strongly recommended to have an intelligent postoperative protocol in place, which is not just helpful but indispensible in a high patient output tertiary care center.

The pathophysiology of development of postoperative EDH is poorly understood and has been largely attributed to low intracranial pressure. Various causes for the same have been put forward like excess CSF drainage, excess blood loss, size of craniotomy and inadvertent loosening of dural hitch sutures. Yu et $\mathrm{al}^{1}$ proposed that the low intracranial pressure as a result of surgery is the most important triggering factor for development of a hematoma. As frequently seen in neurosurgical procedures, the surgical region or the area of interest to surgery is usually located at the highest point during positioning and thus the ipsilateral dura bears a great transmural pressure with the maximum stretching intensity of the bridging vein. This is likely to induce blood vessel rupture resulting in EDH. Since it is the ipsilateral dura which bears the maximum strain, this also means that remote EDH is most likely to occur on the ipsilateral side of surgical region. , 5,7,8 $^{-1}$

CSF release is a neurosurgical procedure in itself and is an integral part of many neurosurgical procedures if not all. Concerning tumor surgeries, it helps in relaxing the brain matter thus providing easier access to pathology. Fukamachi ${ }^{9}$ stated that postoperative EDH may occur due to sudden lowering of intra cranial pressure or rapid drainage of ventricular CSF resulting in brain collapse. Although, none of our patients had a sudden and significant CSF loss as observed in skull base and CSF diversion surgeries, substantial amount of CSF was still drained intra-operatively to aid in the procedure. Excessive CSF drainage leads to intracranial hypotension which further leads to increased trans-luminal venous pressure and subsequent rupture of vessels. This causes an extradural pocket of blood formation which subsequently creates a hydraulic press effect in which the input pressure would be transmitted throughout the hematoma and multiplied. This hypothesis is well and widely accepted and Borkar et $\mathrm{al}^{7}$ stated that it was central to understand 
the pathophysiology of postoperative EDH. The lack of a larger study is explained by the rare nature of occurrence of postoperative remote EDH but such a study is the need of the hour to establish CSF drainage as the prime cause. Also worth mentioning is that replacing CSF loss by instilling saline into the operative cavity before closure might be of no help where remote EDH has started to develop intra-operatively as the site of hematoma is hidden by bone and is far from sight.

Intraoperative blood loss more than $800 \mathrm{ml}$ was put forward as a major causative factor by Kim et al. ${ }^{3}$ They focused primarily on analysis of preoperative hemodynamic factors and intraoperative considerations and didn't analyze the mechanical factors such as CSF drainage. Blood loss more than $800 \mathrm{ml}$ was found to be a significant via both univariate and multivariate analysis and it was proposed that this could be associated with newly developed coagulopathy, loss of coagulation factors, platelet dysfunction and other problems induced by endothelial injury and excessive massive transfusions. This finding needs closer observation and blood loss more than $800 \mathrm{ml}$ as a variable should be put to trial by other studies. This assertion is put forth by the authors themselves too who state that this finding needed more investigation.

Large size of craniotomy as a risk factor has been studied and although no previous data has been published about its relation to incidence of postoperative EDH, craniotomy size larger than $71.53 \mathrm{~cm}^{2}$ was found to be a significant factor. ${ }^{3}$ This could be due to a larger exposure of dura and bone edge which increases the chances of dural detachment. In the same study, when craniectomy was studied as a risk factor over craniotomy, it was not found to be statistically significant. Two of our patients underwent a craniotomy while 1 patient with a final diagnosis of meningioma underwent craniectomy. In our opinion, more important than the craniotomy or craniectomy size is the size of the tumor. This is because low intracranial pressure is a by-product of large tumor surgery. All 3 patients who developed a postoperative remote EDH had a tumor size more than $4 \mathrm{~cm}$ in at least one dimension which is in line with results published by Chung et $\mathrm{al}^{2}$ who observed that out of a total available data of 6 patients regarding size of tumor, 5 patients had a tumor size more than $4 \mathrm{~cm}$ in at least one dimension. Undoubtedly, in most cases the size of tumor dictates the size of craniotomy as a large tumor leads the neurosurgeon to make an appropriate sized craniotomy but mini craniotomies planned along the long axis of tumor also give adequate exposure along the same and thus, in such cases, relying solely on the size of craniotomy over tumor size may result in a false sense of comfort. Also, postoperative remote EDH has also been seen in procedures requiring very small craniotomies like Ventriculo-peritoneal Shunt, which is done via a single burr hole, wherein the generally agreed upon cause is the sudden and significant CSF loss. Authors assert that factors contributing to volume loss play a bigger role than size of craniotomy and whether the former has a stronger association with postoperative remote hematoma over the latter yet remains to be seen via a larger analysis. According to us, other structural characteristics of tumor viz. size, vascularity and proximity to CSF spaces play a vital role in tumor excision and may assume a more significant role.

Inadvertent loosening of dural hitch sutures may precipitate postoperative $\mathrm{EDH}$ and although this entity isn't common, it isn't entirely unknown. No such loosening was observed in any of our cases during the second surgery and the authors thus propose taking hitch sutures at the completion of the surgery rather than immediately after craniotomy. This view is supported by Eroglu et $\mathrm{al}^{10}$, who, in their study challenged prophylactic dural tenting as a dogma in neurosurgery and concluded that fewer complications were indeed observed when dural hitch sutures were placed during post-surgical closure. This timing aids in recognizing a hematoma which might have started to develop intra-operatively as early hitch sutures obliterate the view across the limits of craniotomy. Yet, authors advocate dural hitch sutures at the time of craniotomy if there is significant extra dural bleed intraoperatively at the surgical site, which can be later reinforced with post tumor resection hitch sutures.

Two of our patients developed a hematoma in the frontal region, distant from the site of craniotomy and whether supine nursing during and after the procedure, and the subsequent effect of gravity has a part to play in the same is still a doubt. No data specific to the same has been obtained as yet. However, it seems unlikely as Sinar et $\mathrm{al}^{5}$ reported a posteriorly situated EDH after frontal tumor excision in 2 patients which is in line with findings in our $3^{\text {rd }}$ patient who had a hematoma posterior to surgical region. Other factors like hypertension have also been proposed to play a role in postoperative EDH formation through various studies and while theoretically, hypertension disrupts the hemostatic plug and causes loss of autoregulation ${ }^{9}$, Kim et al in their study could not demonstrate an association between the two.

Various other factors which could not be proven significant were the use of osmotherapy and type and grade of tumor. Osmotherapy doesn't seem to play a significant role in development of pot-operative remote site EDH. This is because the amount of brain relaxation caused by mannitol is far outweighed by relaxation caused by CSF drainage procedures, blood loss greater than 800 $\mathrm{ml}$ and removal of a large sized tumour.

In our series, all 3 patients had a different diagnosis and grades of tumor. This was similar to findings by $\mathrm{Yu}$ et al. who reported remote $\mathrm{EDH}$ in 14 cases, final 


\section{Punia et al}

diagnosis of whom were spread across meningioma, craniopharyngioma, ependymoma and astrocytoma. Furthermore, their WHO gradings were spread equally across the spectrum and included 7 patients with WHO grade-2, four patients of WHO grade- 1 tumors and 5 benign tumors. Thus, it can be confidently asserted that the type and grade of tumor has no bearing on final outcome as far as postoperative remote EDH formation is concerned.

This series have limitations like small sample size, lack of control group and single center series which mandate future studies

\section{Conclusion}

Remote postoperative EDH is a rare and underreported entity; however, neurosurgeons are likely to encounter it at least once in their practice. Excess CSF drainage, volume loss due to tumor size, inadvertent loosening of dural hitch sutures, blood loss more than $800 \mathrm{ml}$ and a large craniotomy are the commonly agreed upon causes. An intelligent protocol for the postoperative scans, placement of dural hitch sutures after large tumor resection and a high index of suspicion may help in avoidance, recognition and if necessary, correction of this complication. Being forewarned is being forearmed.

\section{Conflict of Interest: None \\ Source(s) of support: None}

\section{References}

1. Yu J, Yang F, Cui D, Li Y. Retrospective analysis of 14 cases of remote epidural hematoma as a postoperative complication after intracranial tumor resection. World Journal of Surgical Oncology 2016;14:1-9. https://doi.org/10.1186/s12957-0150754-8
2. Kalfas IH, Little JR. Postoperative hemorrhage: a survey of 4992 intracranial procedures. Neurosurgery 1988;23: 343-7. https://doi.org/10.1227/00006123198809000-00010

3. Kim SH, Lee JH, Joo W, Chough CK, Park HK, Lee KJ et al. Analysis of the risk factors for the development of post-operative extradural hematoma after intracranial surgery. British Journal of Neurosurgery 2015;29(2): 243-8. https://doi.org/10. 3109/02688697.2014.967749

4. Chung JH, Park JS, Park JH, Jeun SS. Remote Postoperative Epidural Hematoma after Brain Tumor Surgery. Brain Tumor Res Treat 2015;3(2): 132-7. https://doi.org/10.14791/btrt.2015.3.2.132

5. Sinar EJ, Lindsay KW. Distant extradural hematoma complicating removal of frontal tumors. J Neurol Neurosurg Psychiatry 1986;49(4): 442-4.

6. Gerlach R, Raabe A, Zimmerman M, Siegemund A, Seifert V. Factor XIII deficiency and postoperative hemorrhage after neurosurgical procedures. Surg Neurol 2000;54: 260-4. https://doi.org/10.1016/ S0090-3019(00)00308-6

7. Borkar SA, Sinha S, Sharma BS. Remote site extradural hematoma. J Clin Neurosci 2009;16: 1097-8.

8. Eom KS, Kim TY, Park JT. Contralateral acute interdural haematoma occurring after burr hole drainage of chronic subdural haematoma. $\mathrm{Br} \mathrm{J}$ Neurosurg 2009;23(2):213-5.

9. Fukamachi A, Koizumi H, Nagaseki Y, Nukui H. Postoperative extradural hematomas: computed tomographic survey of 1105 intracranial operations. Neurosurgery 1986;19(4): 589-93. https://doi. org/10.1227/00006123-198610000-00013.

10. Eroglu U, Zaimoglu M, Sayaci EY, Ugur $\mathrm{HC}$, Attar A, Kahilogullari $\mathrm{G}$ et al. Is placing prophylactic dural tenting sutures a dogma? World Neurosurgery 2021;6:131. http://doi.org/10.1016/j. wneu.2021.06.131. 